\title{
Applications of Cluster Analysis in Diagnostics-Related Problems
}

\author{
V.A. Skormin, Binghamton University, Binghamton, NY, 13902, 607-777-4013, vskormin @binghamton.edu \\ L.J. Popyack, Air Force Research Laboratory, Rome, NY, 13441-5700, 315-330-3010, popyackl@rl.af.mil \\ V.I. Gorodetski, Russian Academy of Sciences, St-Petersburg, Russia, gor@mail.iias.spb.su \\ M.L. Araiza and J.D. Michel, AbTech Corporation, Charlottesville, VA 22911, 804-977-0686, araiza@abtech.com
}

\begin{abstract}
System diagnostics facilitates reliability enhancement and condition-based maintenance of technical systems. Diagnostic problems can be formulated and solved only on the basis of mathematical models reflecting the complex stochastic nature of the failure development process. This process is affected by numerous continuous factors, but its outcome constitutes a random event. These specifics limit the applications of traditional regression models. The concept of a "continuous process with discrete-event output" is introduced, and cluster analysis is employed as a modeling approach facilitating the solution of various diagnostic problems. Important aspects of cluster analysis are the definition of the informativity criterion, selection of "informative subspaces," definition of separating rules, and finally, formulation of a cluster model are presented. Various enhancements of cluster analysis are proposed. A cluster model is utilized for the definition of so-called "probabilistic space" that, in conjunction with Bayes' technique, facilitates the solution of failure prediction problems. Application of cluster models for failure analysis and prediction is illustrated by numerical examples.
\end{abstract}

\section{TABLE OF CONTENTS}

1. INTRODUCTION

2. Cluster ANALYSIS AND DIAGNOSTICS

3. SELECTION OF INFORMATIVE SUBSPACES

4. DEFINITION OF SEPARATING RULES

5. Probabilistic DeCision Making Procedure

6. APPLICATION TO FAILURE ANALYSIS AND PREDICTION OF AN AVIONICS MODULE

\section{INTRODUCTION}

The traditional approach to achieving reliable operation of any technical device (electronics, electrical, electromechanical) is aimed at assuring desired reliability characteristics, such as the average time of normal (nofailure) operation. This approach may be considered acceptable as long as the failures are caused by the factors related to manufacturing, not by the factors representing operational and environmental conditions. Nowadays this approach is not always acceptable. Manufacturers of electronics have achieved a very high degree of reliability of its products. Therefore, manufacturing-related factors on failures are gradually become less significant. The average time of normal operation and other "traditional" reliability characteristics are becoming less important. The main causes of failures of technical systems are traced now to the individual operational and environmental conditions of particular units and to a much lesser extent (due to completely automated manufacturing) individual properties of devices.

Reliable operation of avionics under adverse environmental exposures is crucial for safe and efficient operation of the entire aircraft. While in operation, avionics components are exposed to mechanical vibrations, excessive temperatures, humidity, radiation, etc. These adverse conditions, acting individually and in combination, are known to have cumulative effects leading to avionics performance degradation and failures. Availability of time-stress measurement devices (TSMD) [1] facilitates formulation and solution of important on-line and off-line avionics reliability-related problems. These problems include investigation of the role of various environmental factors in the development of particular failures and combined effects of several factors, reevaluating of probability of failure on the basis of known exposure to particular adverse conditions, as well as development of special types of mathematical models and model-based techniques.

\section{CluSTER ANALYSIS AND DIAGNOSTICS}

System diagnostics is understood as detection, identification and prediction of failures by means of statistical analysis of off- and on-line data. These tasks are always based on mathematical models. Mathematical models describing the physical nature of the failure development process are intended for avionics manufacturers. These models are highly sophisticated and are not suitable for diagnostic, especially on-line, applications. Diagnostics is based on stochastic models representing trend-type effects of various operational and environmental factors that may or may not result in a failure. A failure development process can be viewed as a Continuous Process with Discrete Event Output (CPDEO). The word "continuous" reflects the nature of the recorded environmental variables such as intensity of vibration, humidity, radiation, excessive temperature, etc., and exposure time to these factors. "The discrete event output" refers to the occurrence or non-occurrence of various types of failures as the result of the environmental exposures and operational conditions.

Each observation of a CPDEO is interpreted as a point in a multidimensional factor space representing exposure to adverse conditions and the effect of this exposure, 
$\{X(t), Q(t)\}$

where

$X(t)=\left[x_{1}(t), \quad x_{2}(t), \ldots, x_{n}(t)\right]-$ is a vector of factors representing cumulative exposure to adverse conditions in hours,

$Q(t)=1$ or $Q(t)=0$ - is the integer variable symbolizing the output discrete event ("the device failed", or "normal operation of the device"), and

$t=1,2, \ldots, \mathrm{N}$ - is the observation index.

As a result of this representation, available TSMD data can be divided into two clusters. Analysis of these clusters in the factor space would result in a diagnostic model of the entire class of devices of the same type. This model describes effects of particular factors and combinations of factors on failure development, and allows for the classification of any point of the factor space as belonging to either "normal operation" or "failure" clusters.

Mathematically, such a model could be established as a set of conditions separating the clusters

$$
f_{i}(X)=0, i=1,2, \ldots
$$

Then the decision rule implies checking the value ( 1 or 0 , or "true" or "false") of a predicate defined on the basis of functions (2), and the subsequent "recognition" of the point $X$ in the factor space as a point of one or another cluster.

The choice of functions (2) is dependent upon the geometry and mutual positioning of clusters in the factor space. However, analysis of the above properties in $n$-dimensional space when $n>2$ is impossible, therefore, the problem of choosing a type of classification functions is one of the critical problems in building classification models. The following approach facilitates visualization of the geometry and mutual positioning of clusters by the analysis of projections of clusters on subspaces, strategically selected in the factor space.

The first step of the approach utilizes a special procedure to form a fixed subset of subspaces of the full space of factors. Each subspace is determined by a particular combination of components of the vector of factors. As suggested in [2], the dimension of this combination is assumed to be equal to 2 to make possible the visualization of projections of clusters onto corresponding two-dimensional subspaces (planes). The subspaces included in the fixed subset, and called informative subspaces, are formed based on a special criterion, that provides a measure of informativity of a particular combination of factors for solution of a classification problem. The criterion chosen herein represents the normalized squared distance between a pair of clusters in the corresponding subspace of the factor space. Based on this criterion, a number of two-dimensional subspaces, where the value of the chosen criterion exceeds some margin, are selected.

The next step of the approach includes the definition of separation rules in the selected subspaces and representation of each of these rules as a predicate,

$$
\Phi_{i}=\Phi_{i}\left(f_{i}(X) \# 0\right), i=1,2, \ldots, k
$$

where

$i$ - is the number assigned to a subspace, and

$k$ - is the number of subspaces included in the fixed subset of subspaces.

It could be seen that unlike most traditional classification schemes resulting in one separation rule defined in the subspace of informative factors, our approach utilizes a number of separation rules. Each informative subspace has its own separation rule. From the mathematical point of view, this implies that a general separation surface is being approximated by number of spline-functions (multi facet surfaces). This is one of the main reasons of high efficiency of the approach.

The above predicates are utilized in a set of $2^{k}$ functions of logic, and the entire factor space becomes divided into a number of multi facet regions such that any two regions do not overlap, and the combination of all regions covers the entire factor space. A probabilistic measure is assigned to each of the regions, such that at any point of this region it represents the probability that the point belongs to the cluster "failure." This probability is calculated as a statistical estimate on the basis of experimental data. The approach results in a system of conditional probabilities that facilitate definition of the probability of belonging to the cluster "failure" for any point of the factor space, i.e. defines the probability $P(X / 1)$ for any combination of numerical values of exposures $X$. The utilization of these procedures provides all necessary data for Bayes' formula to compute the probability of failure for a given (current or predicted) value of the exposure vector $X^{*}, P\left(1 / X^{*}\right)$. This constitutes a solution of a prognostic problem.

The general scheme of the approach includes the following steps:

- selection of a set of two dimensional informative subspaces in the factor space, definition of separation rules within each subspace, and predicates of classification,

- definition of a probabilistic space in the factor space,

- definition of a decision making scheme based on the probability of failure of a device for a given value of the vector of adverse exposures.

The first two steps result in the definition of a diagnostic model. The iird step constitutes a model-based decision making. The mathematical model, discussed herein, is applicable to solving such practical reliability related problems as:

- ranking particular environmental conditions as factors responsibile for general and particular types of failures,

- formation of particular groups of environmental conditions and assessment of their combined effects on failures in general and on particular types of failures,

- tracking the dynamics of development of cluster models and their statistical characteristics in the process of obtaining new experimental data,

- justification of the development of devices protecting avionics from adverse environmental conditions, 
- development of the recommendations on the avoidance of the combined effects of adverse conditions.

\section{SELECTION OF INFORMATIVE SUBSPACES}

Informativity analysis is the most computationally intensive task of any classification problem. In our case this is the procedure resulting in a set of two-dimensional informative subspaces. Consider the informativity criterion

$$
\begin{aligned}
& F_{l p}= \\
& \left(\sigma_{l}^{2} \sigma_{p}^{2} N_{l} N_{0}\right)^{-1} \sum_{r=1}^{N_{l}} \sum_{s=1}^{N_{0}}\left\{\left[x_{l}{ }^{l}(r)-x_{l}^{0}(s)\right]^{2}+\left[x_{p}{ }^{l}(r)-x_{p}^{0}(s)\right]^{2}\right\}
\end{aligned}
$$

where

$N_{1}, N_{0}$ - are total number of realizations within the clusters "failure" and "normal operation" respectively, marked by superscripts " 1 " and " 0 ",

$x_{l}^{l}(r), x_{p}^{l}(r)$ - are exposures to the adverse conditions number $l$ and number $q$ in the realization number $r$ belonging to the cluster "failure," $r=1,2, \ldots, N_{1}$ $x_{l}^{0}(s), x_{p}^{0}(s)$ - are exposures to the adverse conditions number $l$ and number $q$ in the realization number $s$ belonging to the cluster "normal operation", $s=1,2, \ldots, N_{0}$ $\sigma_{j}, \sigma_{p}$ - are standard deviations of variables $x_{l}$ and $x_{p}$ estimated over the entire range of experimental data.

The suggested procedure could be implemented through the exhaustive analysis of all low-dimensional subspaces that could be defined in the factor space $X$. It is possible to prove that the algorithm implementing the exhaustive analysis of the measure of informativity (4) for two clusters, $n$ factors, with two-dimensional subspaces has a complexity of

$$
W_{2}=C_{l} N_{0} N_{l} n(n-l)
$$

and with three-dimensional clusters

$$
W_{3}=C_{1} N_{0} N_{1} n(n-1)(n-2) / 2
$$

where

$$
\text { factors. }
$$

$$
C_{J} \text { is a constant, and } n \text { is the total number of }
$$

Consideration of the informativity criteria for one-, two, and three-dimensional subspaces results in a useful recursive formula that could be easily generalized for higher dimensional subspaces

$$
F_{l, p q}=\left(\sigma_{l}^{2} \sigma_{p}^{2} \sigma_{q}^{2}\right)^{-1}\left[\sigma_{l}^{2} \sigma_{p}^{2} F_{l p}+\sigma_{q}^{2} F_{q}\right]
$$

with the complexity

where

$$
W_{3}^{\text {RECURS }}=C_{1} N_{0} N_{1} n+C_{2} n(n-1)(n-2)
$$

$$
C_{2} \ll C_{I} N_{0} N_{l} \text { - is another constant. }
$$

Therefore, one of the ways to enhance the computational efficiency of the considered procedure is to develop more efficient procedures for selecting low-dimensional informative subspaces. Availability of such procedures would allow one to extend the search of informative subspaces to subspaces of any dimension.

The second approach, still under investigation by the authors, implies that the dimension of the factor space can be significantly reduced by performing cluster analysis not in the original space $X$ but in the reduced dimension space of mutually-orthogonal "artificial" variables $Z$ defined by principle component analysis.

\section{DEFINITION OF SEPARATING RULES}

When (ifformative subspaces have been selected, separation rules are to be established on the basis of separating functions (2) defined on each informative subspace such that

$$
f_{i}\left(x_{l}^{0}, x_{p}^{0}\right)>0 \text { and } f_{i}\left(x_{l}^{l}, x_{p}^{l}\right) \# 0
$$

where $l$ and $p$ are indexes of factors included in the $i$-th subspace. Traditionally, in the problem of building separation surfaces it is implied that the clusters are linearly separable. As the experimental results often show, in diagnostic tasks this condition is not met. Therefore, the choice of separation surfaces can be accomplished under less strict conditions and be prompted by the actual topology of cluster projections on informative subspaces, The complexity of this task is significantly reduced by the application of two-dimensional subspaces. Although linear separation surfaces present the simplest solution, much more flexible nonlinear separating surfaces could be suggested as a "general case" solution. The following computer-based procedure for "automatic" definition of separation rules has proven to be quite beneficial.

A separation rule in the space of two variables (factors) $x_{t}$ and $x_{p}$ can be defined on the basis of an ellipse that is justified by most common mutual topologies of two clusters

$$
\begin{gathered}
f_{i}\left(A, x_{6}, x_{p}\right)= \\
\left(x_{l}-a_{l}\right)^{2} / a_{3}^{2}+\left(x_{p}-a_{2}\right)^{2} / a_{4}^{2}+a_{5}\left(x_{l}-a_{1}\right)\left(x_{p}-a_{2}\right) /\left(a_{3} a_{4}\right)-1
\end{gathered}
$$

where

$$
A=\left[a_{1}, a_{2}, a_{3}, a_{4}, a_{5}\right]-\text { is the vector of unknown }
$$
parameters of the ellipse.

Initial values of parameters $A$ can be defined in terms of estimated means and variances of variables $x_{l}^{I}(r), x_{p}{ }^{l}(r)$, $r=1,2, \ldots, N_{l}$. The fine-tuning of these parameters, resulting in the optimal position, orientation, size, and shape of the ellipse was achieved by numerical minimization of the following heuristic criterion

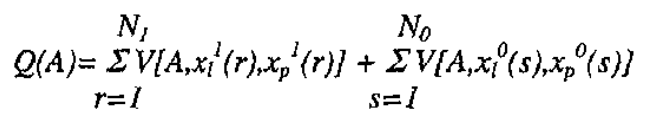

where

$$
V\left[A, x_{l}^{l}(r), x_{p}^{l}(r)\right]=\left[x_{l}^{l}(r)-a_{1}\right]^{2}+\left[x_{p}^{l}(r)-a_{2}\right]^{2}
$$


is the penalty assigned to each point (member) of cluster "failure" that does not satisfy condition $f_{i}\left(A, x_{l}^{l}, x_{p}{ }^{l}\right) \# 0$, otherwise $V\left[A, x_{l}^{I}(r), x_{p}{ }^{l}(r)\right]=0$,

$$
V\left[A, x_{l}^{0}(s), x_{p}^{0}(s)\right]=\left[x_{l}^{0}(s)-a_{l}\right]^{2}+\left[x_{p}^{0}(s)-a_{2}\right]^{2}
$$

is the penalty assigned to each point of cluster "failure" that satisfies condition $f_{i}\left(A, x_{l}^{0}, x_{p}^{0}\right) \# 0$ otherwise $V\left[A, x_{i}^{0}(s), x_{p}^{0}(s)\right]=0$.

The minimization task leading to the estimation of parameters $a_{1}, a_{2}, a_{3}, a_{4}, a_{5}$ of the separating function was performed by using a direct search procedure by Nelder and Mead [ 4].

Note, that in order to accommodate for a different cluster topology, it is suggested to supplement the above analysis by developing separation rules on the basis of ellipses $\varphi_{i}(X)=0, i=1,2, \ldots, k$, such that

$$
\varphi_{i}\left(A, x_{l}^{l}, x_{p}^{l}\right) \# 0 \text { and } \varphi_{i}\left(A, x_{l}^{0}, x_{p}{ }^{0}\right)>0
$$

and optimize their parameters by numerical minimization. Then, for each $i=1,2, . ., k$ the "goodness" of the separating surface $\varphi_{i}(X)=0$ must be compared with the one of $f_{i}(X)=0$ and the best surface shall be selected.

When the task of establishing separation surfaces has been completed, the following logic functions (predicates) can be defined:

$$
\begin{aligned}
& +_{i}(X)=\text { "true" if } f_{i}(X) \neq 0 \\
& +_{i}(X)=" \text { false" if } f_{i}(X)>0, i=1,2, \ldots, k
\end{aligned}
$$

and the following set of conditional probabilities of correct and incorrect classification is established empirically for every $i=1,2, \ldots, k$ :

$$
\begin{aligned}
& P\left(+_{i}(X)=\text { "true" } / X \in \Omega^{\prime}\right)=P_{i}(1 / 1) \\
& P\left(+_{i}(X)=" f a l s e " / X \notin \Omega^{\prime}\right)=P_{i}(O / O) \\
& P\left(+_{i}(X)=\text { "true" } / X \notin \Omega^{\prime}\right)=P_{i}(1 / 0) \\
& P\left(+,(X)=" \text { false" } / X \in \Omega^{\prime}\right)=P_{i}(0 / l)
\end{aligned}
$$

where

$$
\Omega^{\prime} \text { - represents the cluster "failure" }
$$

The empirical probabilities utilized in the above formulas are defined as relative frequencies of the corresponding events in the "true" and "false" regions of classification. Therefore, they satisfy the following equations:

$$
\begin{aligned}
& P_{i}(1 / 1)+P_{i}(0 / 1)=1 \\
& P_{i}(0 / 0)+P_{i}(1 / 0)=1, i=1,2, \ldots, k
\end{aligned}
$$

\section{Probabilistic Decision MAKING ProcedURE}

Consider an application of the developed diagnostic model for failure the assessment of the failure probability $P\left(1 / X^{*}\right)$ given the vector of adverse exposures $X^{*}$. Recall that a set of predicates

$$
+(X)=\left\{+_{l}(X),+_{2}(X), \ldots,++_{k}(X)\right\}
$$

has been established for every informative subspace of the factor space as per (11).

Consider a system of elementary events defined as a set of all conjunctions that can be formed using predicates (13) taken with and without the sign of negation. There is a total of $M=2^{\mathrm{k}}$ of such conjunctions denoted as

$$
\begin{aligned}
& R(X)=\left\{R_{l}(X), R_{2}(X), \ldots, R_{M}(X)\right\}: \\
& R_{J}(X)=+_{1}(X) 1++_{2}(X) 1 \ldots++_{k-1}(X) 1+t_{k}(X) \\
& R_{2}(X)=+_{1}(X) 1++_{2}(X) 1 \ldots++_{k-1}(X) 1 k_{k}(X) \\
& R_{3}(X)=+_{1}(X) 1++_{2}(X) 1 \ldots \quad k=1(X) 1+t_{k}(X) \\
& R_{4}(X)=+_{1}(X) 1++_{2}(X) 1 \ldots \quad k \cdot(X) l \quad k(X) \\
& \ldots \ldots \ldots \ldots \ldots \ldots \ldots \\
& R_{M-l}(X)={ }_{1}(X) 1 \quad{ }_{2}(X) 1 \ldots \quad{ }_{k-1}(X) 1+{ }_{k}(X) \\
& R_{M}(X)={ }_{1}(X) 1{ }_{2}(X) 1 \ldots \quad k-1(X) 1{ }_{k}(X)
\end{aligned}
$$

Each of these conjunctions constitutes a classification predicate and corresponds to a particular region in the factor space. The regions do not overlap, and their sum forms a universal set that in our problem is the entire factor space. Every conjunction (elementary event) can be viewed as a classification predicate, and empirical probabilities of these conjunctions

$$
\begin{aligned}
& P_{h}(1 / 1)=P\left[R_{h}(X)=\text { "true" } / X \in \Omega^{\prime}\right] \\
& P_{h}(1 / 0)=P\left[R_{h}(X)=\text { "true" } / X \notin \Omega^{\prime}\right], h=1,2, \ldots, M
\end{aligned}
$$

can be estimated. The set of probabilities (15) constitutes the "probabilistic space" established in the factor space $X$.

The availability of probabilities (15) allows for the use of Bayes' formula to compute the probability of failure for any point of the factor space $\mathrm{X}^{*}$, subject to the condition

as follows:

$$
\mathrm{R}_{h}\left(X^{*}\right)=\text { "true" }
$$

$$
P\left[1 / R_{h}\left(X^{*}\right)\right]=\frac{P(1) P_{h}(1 / 1)}{P(1) P_{h}(1 / 1)+P(0) P(1 / 0)}
$$

where

$$
P(1), P(2) \text { - are prior probabilities of failure and }
$$
normal operation.

It is obvious that probability of normal operation under the adverse exposures $X^{*}$ is

$$
p_{h}\left[0 / E_{h}\left(X^{*}\right)\right]=1-P\left[1 / E_{h}\left(X^{*}\right)\right]
$$

\section{APPLICATION TO FAILURE ANALYSIS AND PREDICTION OF AN AVIONICS MODULE}

For the purpose of illustration consider the following example utilizing simulated TSMD data comprising status of 50 avionics modules of the same type represented by a binary variable and their exposure time to such adverse environmental conditions. Such a database could be compiled by downloading TSMD records after each sortie and / or by laboratory testing. 
Composition of the database is presented in Table 1 of the Appendix. Application of the developed cluster analysis program resulted in a list of two-dimensional subspaces. The first 20 subspaces, listed according to their informativity, can be found in Table 2 of the Appendix. It could be seen that the list of subspaces could be reduced to first five that are most informative. Printout 1 of the Appendix features computer-generated separating functions (ellipses) of the most informative subspaces and presents estimated probabilities of correct and incorrect classification. Printout 2 of the Appendix presents elementary events defined as conjunctions of the 5 original logic functions with and without negation sign, and the estimated conditional probabilities of these events thus providing the definition of the probabilistic space. Printout 3 of the Appendix shows the distribution of representatives of cluster "failure" (F) and cluster "no failure" (0) in the particular informative subspace (note that " $X$ " represents situations when symbols " $F$ " and " 0 " coincide).

Analysis of the failure process can be performed on the basis of printouts 1 and 2 that feature and rank particular adverse conditions and combinations of adverse conditions resulting in failure.

The defined diagnostic model can be applied for the reevaluation of the probability of failure of the avionics module on the basis of its actual "history of abuse" represented by the vector of adverse exposures,

$$
\begin{gathered}
X^{*}=[1.45,1.42, .65,1.2,1.7,1.44,1.1,0.7,18 ., .5, .2,19 ., \\
1.4, .77, .7,1.5,15 ., 1.9,20.0]
\end{gathered}
$$

Assume that the prior probability of failure is .0001 . This task is easily performed by the developed computer code: first it was determined that appearance of vector $X^{*}$ results in the occurrence of event $E_{29}$ with probabilities

$$
\mathrm{P}(1 / 1)=0.0421, \quad \mathrm{P}(1 / 0)=0.0095 \text {, }
$$

then posterior probability of failure was defined as

$$
(.0001 \times 0.0421) /(.0001 \times 0.0421+.9999 \times 0.0095)=.00044
$$

The posterior probability figure, compared against some marginal values, can facilitate a well-justified maintenance decision.

Testing indicates that the developed software tools are userfriendly and numerically efficient.

\section{REFERENCES}

[1] M. McCallum, L. Popyack, J. Collins, "Environmental Measurement and Recording Techniques Utilizing a Time Stress Measurement Device (TSMD)", RADC, Griffiss AFB, Proceedings - Institute of Environmental Sciences, 1990.

[2] V. Skormin and L, Popyack, "Reliability of Avionics and its "History of Abuse". A Prognostic Techniques", Proceedings of the International Conference on Information and Control (sponsored by US Air Force) in St. Petersburg, Russia, June 5-15, 97

[3] L. Popyack, "Advanced Diagnostic Methods in Avionics", Ph.D. Dissertation, Binghamton University, Binghamton NY, July 1998

[4] Nelder, J.A. and R. Mead "A Simplex Method for Function Minimization", The Computer Journal, 1965

\section{BIOGRAPHY}

Victor A. Skormin is a professor of Electrical Engineering at the Watson School of Engineering, Binghamton University, Binghamton, NY. He holds a Ph.D. degree from Institute of Steel and Alloys, Moscow, USSR (75), and a MS degree from Polytechnic Institute, Alma-Ata, USSR (68). His area of expertise includes conventional and modern control theory, process control, system optimization, mathematical modeling, computer simulation, and system diagnostics. His research efforts included development of laser beam control procedures for laserbased space communication systems, control and optimization for power plant process, diagnostic system for power generators, adaptive motion control systems. $\mathrm{He}$ served as a consultant to a number of companies including Eastman Kodak, Corning Glass Works, General Electric Company, and Air Force Rome Laboratory. He is actively involved in instructional TV and technical lecturing. Dr. Skormin is the recipient of the IEEE Award "For Leadership in Establishing University-Industry Links in Research and Education", the University Award for Graduate Teaching from Binghamton University, and the SUNY Chancellor's Award for Excellence in Teaching. $\mathrm{He}$ is the Editor for Space Systems of the IEEE Transactions on Aerospace and Electronic Systems, and a Senior Member of IEEE.

Leonard J. Popyack is an Electronics Engineer at the Air Force Research Laboratory's (AFRL) Information directorate in Rome, NY. AFRL was formally Rome Laboratory, and Rome Air Development Center (RADC). Popyack joined RADC in 1989 working in the Reliability Sciences area. His current interests include smart sensors, MEMS, signal processing, and prognostic algorithm development. He worked for General Electric in Syracuse, NY from 84-87 developing undersea sonar systems. Popyack holds a Ph.D. (98) and a MS (89) in Electrical Engineering, both from Binghamton University. He is a member if the IEEE since 1983. He enjoys snowmobiling, amateur radio, and hunting in his free time.

Vladimir I. Gorodetski is a Leading Researcher of the St.Petersburg Institute for Information and Automation of the Russian Academy of Science. He holds a MS (60) and a Ph.D. (67) degrees from Military Air Force Engineering Academy of St.Petersburg, and Sc.D. degree (73) from the St.Petersburg State University, Russia. His main areas of research are Optimal Control System Theory, Applied Statistics, Planning, Pattern Recognition and Artificial Intelligence, Spaceship Systems Motion Simulation and Analysis. He served as a Professor of Military Academy of 
Space Engineering, St.Petersburg, and retired in 1992 in the rank of a colonel of Russian AF. Dr. Gorodetski is a member of numerous Russian and international professional organizations. He is the author of over 180 publications including 11 books. His recent research is funded by US AF.

Michael L. Araiza, Vice President, AbTech Corporation, received a BSEE at Rice University in 1980. Mr. Araiza leads many of AbTech's efforts to apply database, expert system, and statistical network technology for diagnostic, prognostic, and signal processing applications. He has been a member if the IEEE since 1985.

Jonathan Michel, Director of Research and Development, AbTech Corporation, received an MS and Ph.D. from University of Virginia, Charlottesville, VA, in 1994 and 1996 in Electrical Engineering, his BS in Computer Engineering (Magna Cum Laude) from the University of Vermont, Burlington, VT, in 1992. Dr. Michel leads AbTech in the development, integration, and validation of advanced statistical algorithms, methodologies, and techniques.
Table 1

\begin{tabular}{|c|c|}
\hline$\#$ & Exposure Factors \& Status \\
\hline 1 & Vibration RMS, $1-2 \mathrm{~g}$ \\
\hline 2 & Vibration RMS, $3-4 \mathrm{~g}$ \\
\hline 3 & Vibration RMS, over $4 \mathrm{~g}$ \\
\hline 4 & Humidity, $20-50 \%$ \\
\hline 5 & Humidity, $70-95 \%$ \\
\hline 6 & Environmental Temperature $-15-0 \mathrm{EC}$ \\
\hline 7 & Environmental Temperature $0-15 \mathrm{EC}$ \\
\hline 8 & Environmental Temperature 50-75EC \\
\hline 9 & Environmental Temperature $76 \cdot 100 \mathrm{EC}$ \\
\hline 10 & Power Supply $.7-.9$ nominal Vdc \\
\hline 11 & Power Supply 1.1 - 1.3 nominal Vdc \\
\hline 12 & Power Supply over 1.3 nominal Vdc \\
\hline 13 & Functional Overload $5-10 \%$ \\
\hline 14 & Functional Overload $11-20 \%$ \\
\hline 15 & Functional Overload $21-30 \%$ \\
\hline 16 & Functional Overload $31-40 \%$ \\
\hline 17 & Functional Overload $41-50 \%$ \\
\hline 18 & Air Pressure $.3-.7$ nominal \\
\hline 19 & Air Pressure $1.1-1.3$ nominal \\
\hline 20 & Status of the module (normal / failure) \\
\hline
\end{tabular}

Table 2

\begin{tabular}{|c|c|c|c|}
\hline$\#$ & $X_{i}$ & $X_{j}$ & $\begin{array}{c}\text { separation } \\
\text { criterion }\end{array}$ \\
\hline 1 & $\mathrm{X}_{4}$ & $\mathrm{X}_{9}$ & 4.822 \\
\hline 2 & $\overrightarrow{\mathrm{X}_{1}}$ & $\overline{X_{9}}$ & 4.784 \\
\hline 3 & $\mathrm{X}_{9}$ & $\mathrm{X}_{17}$ & 4.783 \\
\hline 4 & $\mathrm{X}_{9}$ & $\vec{X}_{14}$ & 4.738 \\
\hline 5 & $\mathrm{X}_{3}$ & $X_{9}$ & 4.726 \\
\hline 6 & $\overrightarrow{X_{9}}$ & $X_{12}$ & 4.725 \\
\hline 7 & $\mathrm{X}_{6}$ & $\mathrm{X}_{9}$ & 4.711 \\
\hline 8 & $\overrightarrow{\mathrm{X}_{9}}$ & $\mathrm{X}_{18}$ & 4.670 \\
\hline 9 & $\widehat{X_{9}}$ & $X_{16}$ & 4.668 \\
\hline 10 & $\overline{X_{9}}$ & $\mathbf{X}_{19}$ & 4.658 \\
\hline 11 & $\mathrm{X}_{5}$ & $\mathrm{X}_{9}$ & 4.614 \\
\hline 12 & $\widehat{\mathrm{X}_{9}}$ & $\mathrm{X}_{15}$ & 4.610 \\
\hline 13 & $\mathrm{X}_{9}$ & $\overline{X_{23}}$ & 4.601 \\
\hline 14 & $X_{9}$ & $\mathrm{X}_{11}$ & 4.588 \\
\hline 15 & $X_{8}$ & $X_{9}$ & 4.580 \\
\hline 15 & $\mathrm{X}_{9}$ & $\mathrm{X}_{10}$ & 4.575 \\
\hline 17 & $\bar{X}_{1}$ & $\mathrm{X}_{4}$ & 4.574 \\
\hline 18 & $\mathrm{X}_{4}$ & $\mathrm{X}_{17}$ & 4.573 \\
\hline 19 & $\mathrm{X}_{2}$ & $\overline{X_{9}}$ & 4.562 \\
\hline 20 & $\overline{X_{7}}$ & $\widetilde{X_{9}}$ & 4.553 \\
\hline
\end{tabular}




\section{Printout 1}

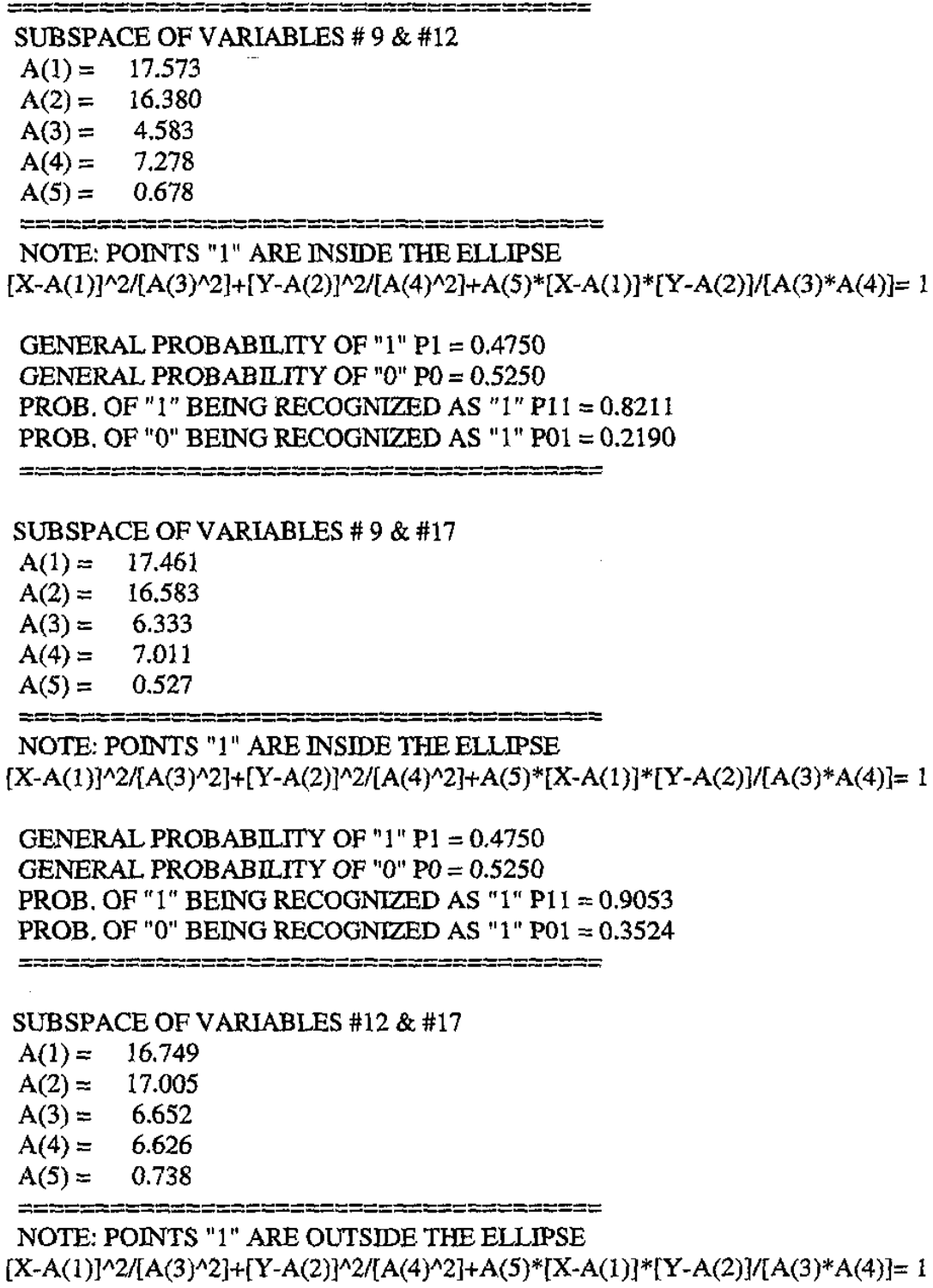

SUBSPACE OF VARIABLES \#12 \& \#17
$A(1)=16.749$
$A(2)=17.005$
$\mathrm{A}(3)=6.652$
$A(4)=6.626$

$A(5)=0.738$

$===$

NOTE: PONNTS "1" ARE OUTSIDE THE ELLIPSE

$[\mathrm{X}-\mathrm{A}(1)]^{\wedge} 2 /\left[\mathrm{A}(3)^{\wedge} 2\right]+[\mathrm{Y}-\mathrm{A}(2)]^{\wedge} 2 /\left[\mathrm{A}(4)^{\wedge} 2\right]+\mathrm{A}(5)^{*}[\mathrm{X}-\mathrm{A}(1)]^{*}[\mathrm{Y}-\mathrm{A}(2)] /\left[\mathrm{A}(3)^{*} \mathrm{~A}(4)\right]=1$

GENERAL PROBABILITY OF "1" P1 $=0.4750$

GENERAL PROBABLITY OF " 0 " $P 0=0.5250$

PROB. OF " 1 " BEING RECOGNTZED AS " 1 " P1 $1=0.9053$

PROB. OF "0" BEING RECOGNIZED AS " 1 " P01 $=0.4095$

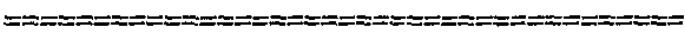


Printout 2

CONDITYONAL PROBABILITIES OF INTEREST:

EVENT 1: $P(1 / 1)=0.0000, \quad P(1 / 0)=0.0762$

EVENT 2: $P(1 / 1)=0.0000, \quad P(1 / 0)=0.0095$

EVENT 3: $P(1 / 1)=0.0000, \quad P(1 / 0)=0.0286$

EVENT 4: $P(1 / 1)=0.0316, \quad P(1 / 0)=0.2381$

EVENT 5: $P(1 / 1)=0.0421, \quad P(1 / 0)=0.0571$

EVENT 6: $\mathrm{P}(1 / 1)=0.0000, \quad \mathrm{P}(1 / 0)=0.0000$

EVENT 7: $P(1 / 1)=0.0000, \quad P(1 / 0)=0.0000$

EVENT 8: $P(1 / 1)=0.0000, \quad P(1 / 0)=0.0095$

EVENT 9: $P(1 / 1)=0.0105, \quad P(1 / 0)=0.0476$

EVENT 10: $P(1 / 1)=0.0000, \quad P(1 / 0)=0.0000$

EVENT 11: $P(1 / 1)=0.0105, \quad P(1 / 0)=0.0667$

EVENT 12: $P(1 / 1)=0.0211, \quad P(1 / 0)=0.0095$

EVENT 13: $P(1 / 1)=0.0526, \quad P(1 / 0)=0.0381$

EVENT 14: $P(1 / 1)=0.0000, \quad P(1 / 0)=0.0000$

EVENT 15: $P(1 / 1)=0.0105, \quad P(1 / 0)=0.0095$

EVENT 16: $P(1 / 1)=0.0000, \quad P(1 / 0)=0.0000$

EVENT 17: $P(1 / 1)=0.0105, \quad P(1 / 0)=0.0286$

EVENT 18: $P(1 / 1)=0.0000, \quad P(1 / 0)=0.0095$

EVENT 19: $P(1 / 1)=0.0105, \quad P(1 / 0)=0.0000$

EVENT 20: $P(1 / 1)=0.0000, \quad P(1 / 0)=0.0000$

EVENT 21: $P(1 / 1)=0.0000, \quad P(1 / 0)=0.0000$

EVENT 22: $P(1 / 1)=0.0000, \quad P(1 / 0)=0.0000$

EVENT 23: $P(1 / 1)=0.0000, \quad P(1 / 0)=0.0095$

EVENT 24: $P(1 / 1)=0.0000, \quad P(1 / 0)=0.0000$

EVENT 25: $P(1 / 1)=0.0000, \quad P(1 / 0)=0.0095$

EVENT 26: $P(1 / 1)=0.0000, \quad P(1 / 0)=0.0190$

EVENT 27: $P(1 / 1)=0.0526, \quad P(1 / 0)=0.0952$

EVENT 28: $P(1 / 1)=0.0105, \quad P(1 / 0)=0.0000$

EVENT 29: $P(1 / 1)=0.0421, \quad P(1 / 0)=0.0095$

EVENT 30: $\mathrm{P}(1 / 1)=0.0105, \quad \mathrm{P}(1 / 0)=0.0000$

EVENT 31: $P(1 / 1)=0.6842, \quad P(1 / 0)=0.0381$

Printout 3

INFORMATIVE SUBSPACE \# 1: $\times(9) \& \times(12)$ CRITERION $=$

$\mathrm{X}(9)(0.8787 \mathrm{E}+01<\cdots \times 0.2126 \mathrm{E}+02$

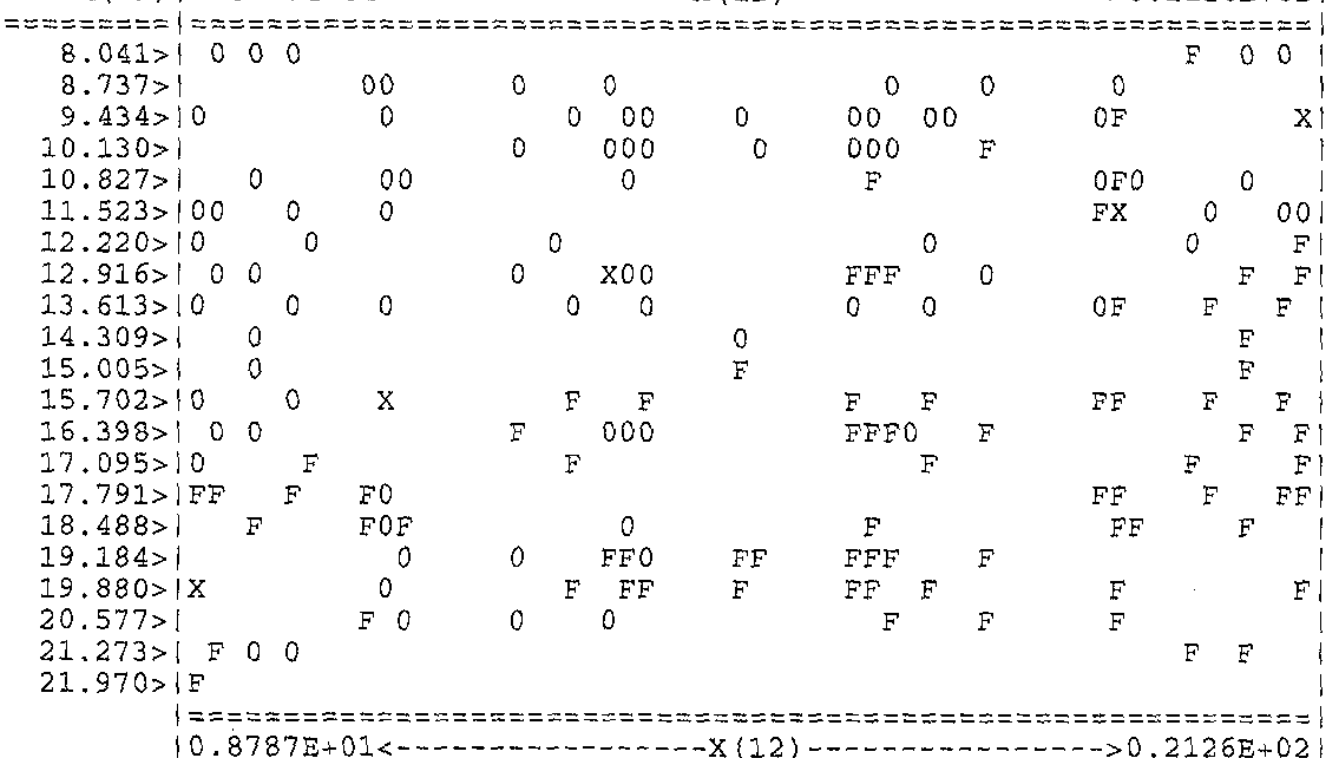

